\title{
Author Index Volume 10 (2011/2012)
}

The issue number is given in front of the pagination

Abu-Soud, H.M., see Fletcher, N.M. (6) 267-275

Ackermann, H., see Tsaur, I. (5) 195-204

Adebisi, I.N., see Pereira, L.H.M. (5) 241-249

Aglietti, M.C., see Mearini, L. (3,4) 117-123

Ali-Fehmi, R., see Fletcher, N.M. (6) 267-275

Altekruse, S.F., see Man-Sun Sy, M.-S. (6) 251-258

Andjelkovic, T., J. Bankovic, Z. Milosevic, J. Stojsic, V. Milinkovic, M. Pesic, S. Ruzdijic and N. Tanic, Concurrent alteration of $p 16$ and PTEN tumor suppressor genes could be considered as potential molecular marker for specific subgroups of NSCLC patients (6) 277-286

Antonacopoulou, A.G., A.E. Kottorou, F.-I.D. Dimitrakopoulos, V. Triantafyllia, S. Marousi, A. Koutras and H.P. Kalofonos, VEGF polymorphisms may be associated with susceptibility to colorectal cancer: A case-control study (5) 213217

Bankovic, J., see Andjelkovic, T. (6) 277-286

Banna, G.L., see Paratore, S. (2) 79-89

Bartling, B., V. Vanhooren, S. Dewaele, C. Libert, H.-S. Hofmann, J. Haerting, S. Nuding, R.-E. Silber, A. Simm and C.C. Chen, Altered desialylated plasma $\mathrm{N}$-glycan profile in patients with non-small cell lung carcinoma $(3,4)$ 145-154

Bartsch, G., see Tsaur, I. (5) 195-204

Bella, V., see Sebova, K. (1) 13-26

Bellia, D., see Paratore, S. (2) 79-89

Belotte, J., see Fletcher, N.M. (6) 267-275

Bigbee, W.L., see Nolen, B.M. (1) 3-12

Bini, V., see Mearini, L. (3,4) 117-123

Blaheta, R.A., see Tsaur, I. (5) 195-204

Blumenschein, Jr., G.R., M. Reck, F. Fossella, D.J. Stewart, C. Lathia and C. Peña, Plasma biomarkers correlating with clinical outcome in a phase II study of sorafenib in advanced NSCLC (6) $287-$ 298
Bravo-Calderón, D.M., D.T. Oliveira, A.N. Marana, S. Nonogaki, A.L. Carvalho and L.P. Kowalski, Prognostic significance of beta-2 adrenergic receptor in oral squamous cell carcinoma (1) 51-59 Buscarino, C., see Paratore, S. (2) 79-89

Cai, Z., see Xu, J. (2) 63-69

Carter, B., see Ramakrishnan, V. (3,4) 185-193

Carvalho, A.L., see Bravo-Calderón, D.M. (1) 51-59

Catlow, J., see McCall, P. (2) 91-99

Cavallaro, S., see Paratore, S. (2) 79-89

Cha, E.-J., see Jeong, P. (5) 205-211

Chen, B., see Niu, H.T. (2) 109-116

Chen, C.C., see Bartling, B. (3,4) 145-154

Chen, C.C., see Ramakrishnan, V. $(3,4)$ 185-193

Chen, J., see Xu, J. (2) 63-69

Chen, J., see Zheng, Y. (2) 71-77

Chen, K., see Ma, S. $(3,4)$ 155-162

Cho, I.-C., see Jeong, P. (5) 205-211

Choi, S., see Nolen, B.M. (1) 3-12

Chung, K., N. Nishiyama, S. Yamano, H. Komatsu, S. Hanada, M. Wei, H. Wanibuchi, S. Suehiro and A. Kakehashi, Serum AGR2 as an early diagnostic and postoperative prognostic biomarker of human lung adenocarcinoma (2) 101-107

Corbett, S., see Topilow, A.A. (1) 27-33

Cui, L., see Zheng, Y. (2) 71-77

Cunsolo, R., see Paratore, S. (2) 79-89

Dacic, S., see Nolen, B.M. (1) 3-12

D’Arrigo, M., see Paratore, S. (2) 79-89

Davis, J.M., see Topilow, A.A. (1) 27-33

de las Rivas, J., see Folio, C. (1) 35-41

Dewaele, S., see Bartling, B. $(3,4)$ 145-154

Dey, S., see Gill, K. $(3,4) 125-134$

Diamond, M.P., see Fletcher, N.M. (6) 267-275

Dimitrakopoulos, F.-I.D., see Antonacopoulou, A.G. (5) 213-217

Dong, Q., see Niu, H.T. (2) 109-116 
Duncan, R., see Pereira, L.H.M. (5) 241-249

Edwards, J., see McCall, P. (2) 91-99

Elgaaied, A.B.A., see Ouerhani, S. (6) 259-266

Endo, S., see Misawa, K. $(3,4)$ 135-144

Fletcher, N.M., Z. Jiang, R. Ali-Fehmi, N.K. Levin, J. Belotte, M.A. Tainsky, M.P. Diamond, H.M. Abu-Soud and G.M. Saed, Myeloperoxidase and free iron levels: Potential biomarkers for early detection and prognosis of ovarian cancer (6) 267275

Folio, C., M. Zalacain, C. Zandueta, C. Ormazábal, L. Sierrasesúmaga, M.S. Julián, J. de las Rivas, G. Toledo, F. Lecanda and A. Patiño-García, Cortactin (CTTN) overexpression in osteosarcoma correlates with advanced stage and reduced survival (1) 35-41

Fossella, F., see Blumenschein, Jr., G.R. (6) 287-298

Franzmann, E.J., see Pereira, L.H.M. (5) 241-249

Fridrichova, I., see Sebova, K. (1) 13-26

Galbavy, S., see Sebova, K. (1) 13-26

Gasser, M., see Tsaur, I. (5) 195-204

Gill, K., B.K. Mohanti, A.K. Singh, B. Mishra and S. Dey, The over expression of cathelicidin peptide LL37 in head and neck squamous cell carcinoma: The peptide marker for the prognosis of cancer $(3,4)$ 125-134

Gilvarg, C., see Topilow, A.A. (1) 27-33

Gong, G.-H., see Ha, S.-A. (5) 219-231

Goodman, M.T., see Man-Sun Sy, M.-S. (6) 251-258

Goodwin, W.J., see Pereira, L.H.M. (5) 241-249

Guo, H., see Xu, Q. (5) 233-239

Guo, J., see Zheng, Y. (2) 71-77

Ha, S.-A., Y.S. Lee, H.K. Kim, J. Yoo, S. Kim, G.H. Gong, Y.K. Lee and J.W. Kim, The prognostic potential of keratin 18 in breast cancer associated with tumor dedifferentiation, and the loss of estrogen and progesterone receptors (5) 219-231

Ha, Y.S., see Jeong, P. (5) 205-211

Haerting, J., see Bartling, B. $(3,4) 145-154$

Haferkamp, A., see Tsaur, I. (5) 195-204

Hakamada, K., see Misawa, K. (3,4) 135-144

Hanada, S., see Chung, K. (2) 101-107

He, J., see Xu, J. (2) 63-69

Hernandez, B.Y., see Man-Sun Sy, M.-S. (6) 251-258

Hewitt, S.M., see Man-Sun Sy, M.-S. (6) 251-258

Hofmann, H.-S., see Bartling, B. (3,4) 145-154

Honda, K., see Yokomizo, A. $(3,4)$ 175-183
Hu, J.J., see Pereira, L.H.M. (5) 241-249

Huesch, T., see Tsaur, I. (5) 195-204

Huo, M., see Zheng, Y. (2) 71-77

Iemmolo, R., see Paratore, S. (2) 79-89

Imai, A., see Misawa, K. (3,4) 135-144

Jeong, P., Y.S. Ha, J.S. Kim, I.-C. Cho, W.T. Kim, Y.-J. Kim, I.Y. Kim, S.-J. Yun, S.-C. Lee, E.-J. Cha and W.-J. Kim, Runt-related transcription factor 3 methylation as a possible prognosticator in muscle-invasive bladder cancer (5) 205-211

Jiang, Z., see Fletcher, N.M. (6) 267-275

Julián, M.S., see Folio, C. (1) 35-41

Kajabova, V., see Sebova, K. (1) 13-26

Kajo, K., see Sebova, K. (1) 13-26

Kakehashi, A., see Chung, K. (2) 101-107

Kalofonos, H.P., see Antonacopoulou, A.G. (5) 213217

Kanai, T., see Yokomizo, A. $(3,4)$ 175-183

Kanazawa, T., see Misawa, K. $(3,4)$ 135-144

Kim, H.K., see Ha, S.-A. (5) 219-231

Kim, I.Y., see Jeong, P. (5) 205-211

Kim, J.S., see Jeong, P. (5) 205-211

Kim, J.W., see Ha, S.-A. (5) 219-231

Kim, S., see Ha, S.-A. (5) 219-231

Kim, W.-J., see Jeong, P. (5) 205-211

Kim, W.T., see Jeong, P. (5) 205-211

Kim, Y.-J., see Jeong, P. (5) 205-211

Koay, D.C., see Ramakrishnan, V. (3,4) 185-193

Komatsu, H., see Chung, K. (2) 101-107

Kottorou, A.E., see Antonacopoulou, A.G. (5) 213-217

Koutras, A., see Antonacopoulou, A.G. (5) 213-217

Kowalski, L.P., see Bravo-Calderón, D.M. (1) 51-59

Krivulcik, T., see Sebova, K. (1) 13-26

Kumar, C.A., see Sawhney, H. (1) 43-49

Kurosch, M., see Tsaur, I. (5) 195-204

Kushwaha, D., see Ramakrishnan, V. (3,4) 185-193

Langmead, C.J., see Nolen, B.M. (1) 3-12

Lasabova, Z., see Sebova, K. (1) 13-26

Lathia, C., see Blumenschein, Jr., G.R. (6) 287-298

Lazariotou, M., see Tsaur, I. (5) 195-204

Lecanda, F., see Folio, C. (1) 35-41

Lee, S.-C., see Jeong, P. (5) 205-211

Lee, Y.K., see Ha, S.-A. (5) 219-231

Lee, Y.S., see Ha, S.-A. (5) 219-231

Levin, N.K., see Fletcher, N.M. (6) 267-275

Li, C., see Man-Sun Sy, M.-S. (6) 251-258

Li, J., see Xu, Q. (5) 233-239 
Liang, Z., see Xu, J. (2) 63-69

Libert, C., see Bartling, B. (3,4) 145-154

Lipari, H., see Paratore, S. (2) 79-89

Lokeshwar, V.B., see Pereira, L.H.M. (5) 241-249

Lokshin, A.E., see Nolen, B.M. (1) 3-12

Lomakin, A., see Nolen, B.M. (1) 3-12

Lou, Y., see Zheng, Y. (2) 71-77

Lucenti, L., see Paratore, S. (2) 79-89

Luo, X., see Xu, J. (2) 63-69

Lynch, C.F., see Man-Sun Sy, M.-S. (6) 251-258

Ma, S., L. Shen, N. Qian and K. Chen, The prognostic values of CA125, CA19.9, NSE, AND SCC for stage I NSCLC are limited $(3,4)$ 155-162

Makarevic, J., see Tsaur, I. (5) 195-204

Man-Sun Sy, M.-S., S.F. Altekruse, C. Li, C.F. Lynch, M.T. Goodman, B.Y. Hernandez, L. Zhou, M.S. Saber, S.M. Hewitt and W. Xin, Association of prion protein expression with pancreatic adenocarcinoma survival in the SEER residual tissue repository (6) 251-258

Mao, Y., see Ramakrishnan, V. (3,4) 185-193

Marana, A.N., see Bravo-Calderón, D.M. (1) 51-59

Marousi, S., see Antonacopoulou, A.G. (5) 213-217

Marrangoni, A., see Nolen, B.M. (1) 3-12

Matsubara, J., see Yokomizo, A. (3,4) 175-183

McArdle, P.A., see McCall, P. (2) 91-99

McCall, P., J. Catlow, P.A. McArdle, D.C. McMillan and J. Edwards, Tumoral C-reactive protein and nuclear factor kappa-B expression are associated with clinical outcome in patients with prostate cancer (2) 91-99

McMillan, D.C., see McCall, P. (2) 91-99

Mearini, L., A. Zucchi, E. Scarponi, E. Nunzi, M.C. Aglietti, V. Bini and M. Porena, Correlation between age and Chromogranin A determination in prostate diseases $(3,4)$ 117-123

Milinkovic, V., see Andjelkovic, T. (6) 277-286

Milosevic, Z., see Andjelkovic, T. (6) 277-286

Mineta, H., see Misawa, K. (3,4) 135-144

Misawa, K., T. Kanazawa, Y. Misawa, A. Imai, S. Endo, K. Hakamada and H. Mineta, Hypermethylation of collagen $\alpha 2$ (I) gene (COL1A2) is an independent predictor of survival in head and neck cancer $(3,4)$ 135-144

Misawa, Y., see Misawa, K. (3,4) 135-144

Mishra, B., see Gill, K. (3,4) 125-134

Mohanti, B.K., see Gill, K. $(3,4)$ 125-134

Naito, S., see Yokomizo, A. $(3,4)$ 175-183

$\mathrm{Ng}, \mathrm{K}$., see Ramakrishnan, V. $(3,4) 185-193$
Nishiyama, N., see Chung, K. (2) 101-107

Niu, H.T., C.M. Yang, B. Chen and Q. Dong, Biomarker research and some deduction in superficial bladder cancer cells combined with corresponding stroma (2) 109-116

Noack, A., see Tsaur, I. (5) 195-204

Nolen, B.M., C.J. Langmead, S. Choi, A. Lomakin, A. Marrangoni, W.L. Bigbee, J.L. Weissfeld, D.O. Wilson, S. Dacic, J.M. Siegfried and A.E. Lokshin, Serum biomarker profiles as diagnostic tools in lung cancer (1) 3-12

Nonogaki, S., see Bravo-Calderón, D.M. (1) 51-59

Nuding, S., see Bartling, B. (3,4) 145-154

Nunzi, E., see Mearini, L. $(3,4)$ 117-123

Oliveira, D.T., see Bravo-Calderón, D.M. (1) 51-59

Ono, M., see Yokomizo, A. $(3,4)$ 175-183

Oppermann, E., see Tsaur, I. (5) 195-204

Ormazábal, C., see Folio, C. (1) 35-41

Ouerhani, S. and A.B.A. Elgaaied, The mutational spectrum of HRAS, KRAS, NRAS and FGFR3 genes in bladder cancer (6) 259-266

Paratore, S., G.L. Banna, M. D’Arrigo, S. Saita, R. Iemmolo, L. Lucenti, D. Bellia, H. Lipari, C. Buscarino, R. Cunsolo and S. Cavallaro, CXCR4 and CXCL12 immunoreactivities differentiate primary non-small-cell lung cancer with or without brain metastases (2) 79-89

Patiño-García, A., see Folio, C. (1) 35-41

Peña, C., see Blumenschein, Jr., G.R. (6) 287-298

Pereira, L.H.M., I.N. Adebisi, A. Perez, M. Wiebel, I. Reis, R. Duncan, W.J. Goodwin, J.J. Hu, V.B. Lokeshwar and E.J. Franzmann, Salivary markers and risk factor data: A multivariate modeling approach for head and neck squamous cell carcinoma detection (5) 241-249

Perez, A., see Pereira, L.H.M. (5) 241-249

Pesic, M., see Andjelkovic, T. (6) 277-286

Porena, M., see Mearini, L. $(3,4)$ 117-123

Qian, N., see Ma, S. (3,4) 155-162

Ramakrishnan, V., D. Kushwaha, D.C. Koay, H. Reddy, Y. Mao, L. Zhou, K. Ng, P. Zinn, B. Carter and C.C. Chen, Post-transcriptional regulation of $\mathrm{O}^{6}$ methylguanine-DNA methyltransferase MGMT in glioblastomas $(3,4)$ 185-193

Reck, M., see Blumenschein, Jr., G.R. (6) 287-298

Reddy, H., see Ramakrishnan, V. $(3,4)$ 185-193

Reis, I., see Pereira, L.H.M. (5) 241-249 
Ren-Heidenreich, L., see Xu, J. (2) 63-69

Rodriguez-Canales, J., see Rosado, L.A.R. $(3,4) 163-$ 173

Rosado, L.A.R., J. Rodriguez-Canales and B. Zhang, Association of D4-GDI expression with breast cancer progression $(3,4)$ 163-173

Ruzdijic, S., see Andjelkovic, T. (6) 277-286

Saber, M.S., see Man-Sun Sy, M.-S. (6) 251-258

Saed, G.M., see Fletcher, N.M. (6) 267-275

Saita, S., see Paratore, S. (2) 79-89

Sakuma, T., see Yokomizo, A. $(3,4)$ 175-183

Sawhney, H. and C.A. Kumar, Correlation of serum biomarkers (TSA \& LSA) and epithelial dysplasia in early diagnosis of oral precancer and oral cancer (1) 43-49

Scarponi, E., see Mearini, L. $(3,4)$ 117-123

Schmitt, L., see Tsaur, I. (5) 195-204

Sebova, K., I. Zmetakova, V. Bella, K. Kajo, I. Stankovicova, V. Kajabova, T. Krivulcik, Z. Lasabova, M. Tomka, S. Galbavy and I. Fridrichova, RASSF1A and $C D H 1$ hypermethylation as potential epimarkers in breast cancer (1) 13-26

Shen, L., see Ma, S. (3,4) 155-162

Siegfried, J.M., see Nolen, B.M. (1) 3-12

Sierrasesúmaga, L., see Folio, C. (1) 35-41

Silber, R.-E., see Bartling, B. (3,4) 145-154

Simm, A., see Bartling, B. (3,4) 145-154

Singh, A.K., see Gill, K. $(3,4)$ 125-134

Stankovicova, I., see Sebova, K. (1) 13-26

Stewart, D.J., see Blumenschein, Jr., G.R. (6) 287-298

Stojsic, J., see Andjelkovic, T. (6) 277-286

Suehiro, S., see Chung, K. (2) 101-107

Sun, W., see Zheng, Y. (2) 71-77

Tainsky, M.A., see Fletcher, N.M. (6) 267-275

Takakura, M., see Yokomizo, A. (3,4) 175-183

Tang, D., see Topilow, A.A. (1) 27-33

Tanic, N., see Andjelkovic, T. (6) 277-286

Toledo, G., see Folio, C. (1) 35-41

Tomka, M., see Sebova, K. (1) 13-26

Topilow, A.A., J.M. Davis, J.J. Vernick, D. Tang, S. Corbett, T. Veltman, S.J. Wagner and C. Gilvarg, Confirmation of a potential biomarker for earlystage pancreatic cancer (1) 27-33

Triantafyllia, V., see Antonacopoulou, A.G. (5) 213217

Tsaur, I., A. Noack, A.M. Waaga-Gasser, J. Makarevic, L. Schmitt, M. Kurosch, T. Huesch, C. Wiesner, S. Wedel, G. Bartsch, H. Ackermann, E. Oppermann, M. Lazariotou, M. Gasser, A. Haferkamp and R.A. Blaheta, Chemokines involved in tumor promotion and dissemination in patients with renal cell cancer (5) 195-204

Vanhooren, V., see Bartling, B. (3,4) 145-154

Veltman, T., see Topilow, A.A. (1) 27-33

Vernick, J.J., see Topilow, A.A. (1) 27-33

Waaga-Gasser, A.M., see Tsaur, I. (5) 195-204

Wagner, S.J., see Topilow, A.A. (1) 27-33

Wanibuchi, H., see Chung, K. (2) 101-107

Wedel, S., see Tsaur, I. (5) 195-204

Wei, M., see Chung, K. (2) 101-107

Weissfeld, J.L., see Nolen, B.M. (1) 3-12

Wiebel, M., see Pereira, L.H.M. (5) 241-249

Wiesner, C., see Tsaur, I. (5) 195-204

Wilson, D.O., see Nolen, B.M. (1) 3-12

Xin, W., see Man-Sun Sy, M.-S. (6) 251-258

Xu, J., J. He, H. Yang, X. Luo, Z. Liang, J. Chen, Z. Cai, J. $\mathrm{Xu}$ and L. Ren-Heidenreich, Somatic mutation analysis of EGFR, KRAS, BRAF and PIK3CA in 861 patients with non-small cell lung cancer (2) 63-69

Xu, J., see Xu, J. (2) 63-69

Xu, Q., B. Yuan, F. Xue, L. Zhang, J. Li, H. Guo and T. Yue, OPN gene polymorphisms are associated with susceptibility and clinicopatholigical characteristics of cervical cancer in a Chinese cohort (5) 233-239

Xue, F., see Xu, Q. (5) 233-239

Yamada, T., see Yokomizo, A. $(3,4)$ 175-183

Yamano, S., see Chung, K. (2) 101-107

Yang, C.M., see Niu, H.T. (2) 109-116

Yang, H., see Xu, J. (2) 63-69

Yokomizo, A., M. Takakura, Y. Kanai, T. Sakuma, J. Matsubara, K. Honda, S. Naito, T. Yamada and M. Ono, Use of quantitative shotgun proteomics to identify fibronectin 1 as a potential plasma biomarker for clear cell carcinoma of the kidney $(3,4) 175-183$

Yoo, J., see Ha, S.-A. (5) 219-231

Yuan, B., see Xu, Q. (5) 233-239

Yuan, X., see Zheng, Y. (2) 71-77

Yue, T., see Xu, Q. (5) 233-239

Yun, S.-J., see Jeong, P. (5) 205-211

Zalacain, M., see Folio, C. (1) 35-41

Zandueta, C., see Folio, C. (1) 35-41

Zhang, B., see Rosado, L.A.R. (3,4) 163-173 
Zhang, L., see Xu, Q. (5) 233-239

Zheng, Y., L. Cui, W. Sun, H. Zhou, X. Yuan, M. Huo, J. Chen, Y. Lou and J. Guo, MicroRNA-21 is a new marker of circulating tumor cells in gastric cancer patients (2) 71-77

Zhou, H., see Zheng, Y. (2) 71-77
Zhou, L., see Man-Sun Sy, M.-S. (6) 251-258

Zhou, L., see Ramakrishnan, V. $(3,4)$ 185-193

Zinn, P., see Ramakrishnan, V. $(3,4)$ 185-193

Zmetakova, I., see Sebova, K. (1) 13-26

Zucchi, A., see Mearini, L. $(3,4)$ 117-123 